\title{
TERAPIA INALATÓRIA EM VENTILAÇÃO NÃO-INVASIVA EM PACIENTES COM DOENÇA PULMONAR OBSTRUTIVA CRÔNICA
}

\author{
INHALATION THERAPY DURING NONINVASIVE \\ VENTILATION IN PATIENTS WITH CHRONIC OBSTRUCTIVE \\ PULMONARY DISEASE
}

\author{
Kathleen Asturian"1, Maria Angélica Pires Ferreira², Diogo Pilger ${ }^{1}$ \\ RESUMO
}

O objetivo deste estudo é descrever os aspectos técnicos e cuidados a serem observados durante a administração de medicamentos inalatórios contidos em inalador pressurizado ( $\mathrm{pMDI}$ ) em pacientes com doença pulmonar obstrutiva crônica em ventilação não-invasiva (VNI). Trata-se de uma revisão integrativa elaborada em quatro fases: (1) busca na literatura; (2) consulta às sociedades médicas; (3) pesquisa em bulas e monografias dos fármacos inalatórios; e (4) contato com empresas de ventiladores, medicamentos e espaçadores. A busca aos estudos foi conduzida a partir de palavras-chaves e restringida a publicações até 31 de dezembro de 2019, nos idiomas português e inglês. Foram selecionados 9 artigos, sendo 1 estudo primário, 6 estudos de revisão e 2 estudos em modelos experimentais de ventilação. Foram incluídas 2 diretrizes a partir da consulta às sociedades médicas e das 8 empresas contatas, quatro fizeram recomendações quanto à técnica inalatória. Não foram encontradas informações sobre a administração dos fármacos em VNI nas bulas e monografias. Recomenda-se atentar para as medidas que podem aumentar a deposição pulmonar dos fármacos inalatórios, como uso de aerocâmara com pMDI, minimização de escape indesejável de ar, sincronia entre paciente-ventilador, disparo do jato na fase inspiratória e inserção de porta de vazamento na máscara ou circuito.

Palavras-chaves: Ventilação não invasiva; Administração inalatória; Inaladores de dose calibrada; Espaçadores para inalação

\section{ABSTRACT}

The aim of this study is to describe the technical aspects and cautions to be observed during the administration of inhaled medications contained in a pressurized inhaler (pMDI) in patients with chronic obstructive pulmonary disease on noninvasive ventilation. This integrative review consisted of 4 phases: (1) search in the literature; (2) consultation with medical societies; (3) research on package inserts and monographs of inhaled drugs; and (4) contact with ventilator, medication and spacer companies. The search for studies was based on keywords and restricted to articles published until December 31, 2019, written in Portuguese and English. Nine articles were selected, including 1 primary study, 6 review studies and 2 studies on experimental ventilation models. Two guidelines were included from the consultation with medical societies, and of the 8 companies contacted, 4 made recommendations regarding the inhalation technique. No information was found on the administration of noninvasive ventilation drugs in package inserts and monographs. Attention should be given to measures that increase the pulmonary deposition of inhaled drugs, such as the use of an air chamber with pMDI, minimization of undesirable air leakage, patient-ventilator synchronization, jet firing in the inspiratory phase and insertion of a leak port in the mask or circuit.

Keywords: Noninvasive ventilation; Inhalation; Metered dose inhalers; Inhalation spacers

Clin Biomed Res. 2021;41(4):347-353

1 Faculdade de Farmácia, Universidade Federal do Rio Grande do Sul. Porto Alegre, RS, Brasil.

2 Comissão de Medicamentos, Hospital de Clínicas de Porto Alegre. Porto Alegre, RS, Brasil.

Autor correspondente: Kathleen Asturian asturiank@gmail.com Faculdade de Farmácia, Universidade Federal do Rio Grande do Sul

Rua São Luís, 154 90620-170, Porto Alegre, RS, Brasil. 
Asturian et al.

\section{INTRODUÇÃO}

A Doença Pulmonar Obstrutiva Crônica (DPOC) é uma condição comum, evitável e tratável, caracterizada por sintomas respiratórios persistentes e limitação do fluxo aéreo devido a anormalidades das vias aéreas e/ou alveolares geralmente causadas por exposição quantitativa e temporalmente significativa a partículas ou gases nocivos ${ }^{1}$. A administração de medicamentos broncodilatadores e anti-inflamatórios por via inalatória é base do tratamento farmacológico de pessoas com DPOC sendo usada tanto em pacientes ambulatoriais, quanto no tratamento de exacerbações ${ }^{2}$.

Apesar de ser comumente utilizados por pacientes com doenças respiratórias crônicas, a complexidade da técnica inalatória suscita dúvidas em todos os contextos assistenciais, tanto por parte dos pacientes como da equipe assistencial. De fato, vários estudos avaliando a adequação da técnica inalatória identificam significativo desconhecimento e alta frequência de erros por parte de profissionais de saúde, mesmo em ambiente hospitalar ${ }^{3-5}$.

Uma das situações em que se faz necessário um maior conhecimento quanto a administração inalatória de fármacos é durante a ventilação mecânica nãoinvasiva (VNI), método de suporte ventilatório que tem sido cada vez mais indicado no tratamento de exacerbações da DPOC, visto sua capacidade de diminuir a fadiga respiratória, realizar a manutenção das trocas gasosas e ainda diminuir o risco de intubação endotraqueal ${ }^{6-8}$.

O objetivo desse estudo é descrever os principais aspectos técnicos e cuidados a serem observados durante a inalação de fármacos contidos em inalador pressurizado (pressurized metered dose inhaler-pMDI) a pacientes em suporte ventilatório não-invasivo, bem como, elaborar recomendações que possam auxiliar na otimização da correta técnica inalatória neste contexto especial.

\section{MÉTODOS}

Trata-se de uma revisão integrativa de literatura, elaborada em quatro fases, guiadas com base na elaboração da seguinte pergunta norteadora: "Quais são as formas de administração de fármacos inalatórios contidos em pMDI a pacientes em ventilação mecânica não-invasiva? Quais os fatores clínicos e parâmetros do aparelho que afetam a entrega do aerossol às vias aéreas inferiores em pacientes com DPOC?"

Para a pesquisa dos estudos na literatura, realizou-se uma busca nas seguintes bases de dados: Pubmed, Lilacs, Embase e Cochrane. Foram utilizados para a busca dos estudos os seguintes descritores e suas combinações nas línguas portuguesa e inglesa: "ventilação não-invasiva", "suporte ventilatório", "técnica inalatória" e "administração inalatória", restringidas a publicações até 31 de dezembro de 2019. Os critérios de inclusão foram: artigos de revisão (de qualquer tipo), estudos primários e estudos com modelos experimentais de ventilação utilizando a técnica inalatória com pMDI a pacientes com DPOC ou não. Foram excluídos os estudos com técnica inalatória em cânula nasal de alto fluxo, traqueostomia, terapia inalatória com nebulizadores e estudos em população pediátrica.

A análise dos estudos selecionados foi extraída conforme instrumento de coleta de dados recomendado para revisões integrativas e sintetizada em tabelas utilizando o softawe Excel ${ }^{\circledR 9}$. A partir de uma análise qualitativa de conteúdos foram identificadas as principais formas de administração, os problemas relatados e as recomendações com vistas a evitá-los.

Realizou-se uma busca adicional por diretrizes, consensos e protocolos clínicos em sítios eletrônicos de cinco sociedades médicas: Sociedade Brasileira de Pneumologia e Tisiologia; American Thoracic Society; European Respiratory Society; Canadian Thoracic Society; e The Thoracic Society of Australia and New Zealand, além da diretriz da Global Initiative for Chronic Obstructive Lung Disease (GOLD) ${ }^{1}$.

Uma consulta às bulas dos medicamentos inalatórios de referência foi realizada: salbutamol $100 \mu \mathrm{g}$; fumarato de formoterol di-hidratado/budesonida $6 / 100$ e 6/200 $\mu$ g; e brometo de ipratrópio/bromidrato de fenoterol 0,020 mg/0,050 mg - todos esses usados como terapia farmacológica na DPOC ${ }^{2}$. Realizou-se também consultas às monografias dos fármacos nas bases de dados Micromedex ${ }^{\mathrm{TM}}$ e UptoDate ${ }^{\mathrm{TM}}$.

Por fim, contatou-se três empresas brasileiras fornecedoras de aparelhos de suporte ventilatório, três indústrias farmacêuticas produtoras de medicamentos inalatórios e duas empresas estrangeiras produtoras de aerocâmaras especiais através do Serviço de Atendimento ao Consumidor (SAC).

\section{RESULTADOS}

Um total de 669 artigos foi encontrado no somatório das bases de dados consultadas. Após a remoção das duplicatas e aplicação dos critérios de inclusão e exclusão, por meio da leitura de títulos e resumos, foram selecionadas para análise 9 publicações ${ }^{10-18}$ sendo 1 estudo primário, 6 estudos de revisão e 2 estudos em modelos experimentais.

O medicamento de estudo em todas as publicações foi o broncodilatador salbutamol $100 \mu \mathrm{g}$ e três marcas de aerocâmaras específicas para ventilação mecânica foram usadas para os testes: Aerovent ${ }^{\circledR}$ (Monaghan Medical, Plattsburgh, NY); AeroChamber-MV ${ }^{\circledR}$ (TrudellMedical International, Canadá); e Volumatic Spacer Device ${ }^{\circledR}$ (Allen and Hanbury, Greenford, UK). A seguir, a descrição dos fatores que afetam a aerossolterapia nessa condição especial conforme os estudos selecionados. 


\section{FATORES RELACIONADOS AO INALADOR PRESSURIZADO}

\section{Tipo de propelente}

O inalador pressurizado contém uma mistura de propelentes, surfactantes, flavorizantes e conservantes, além da substância ativa. Esta mistura permanece contida em um recipiente de alumínio onde há uma válvula de medição e uma haste que se encaixa a um recipiente plástico em formato de $L$, popularmente chamado de "bombinha".

O hidrofluoralcano (HFA) é um propelente seguro e eficaz, reconhecido pela União Europeia e, atualmente, utilizado nos inaladores pressurizados. Ele foi substituído em 1995, pelo até então usado, clorofluorcarbono (CFC), que apresentava efeitos deletérios à camada de ozônio. Quando foi realizada a transição de um propelente pelo outro, o HFA apresentou incompatibilidade com os surfactantes e, por isso, os pMDls precisaram ser reformulados, a fim de adquirir estabilidade entre os excipientes. Essa reformulação gerou um spray aerossol mais fino, acarretando maior deposição pulmonar quando comparado com os antigos aerossóis produzidos com CFC. O tipo de substância ativa e o diâmetro de partícula, assim como a utilização de aerocâmara, influenciam na deposição pulmonar ${ }^{18}$.

\section{Uso de aerocâmara}

O uso de pMDI deve ser incorporado a um segundo dispositivo: aerocâmara (espaçador), especialmente em situações em que o uso correto do pMDI é improvável. Para ventilação mecânica invasiva (VMI), vários pesquisadores têm mostrado que o emprego de uma aerocâmara acoplado ao inalador pressurizado aumenta de 4 a 6 vezes a oferta de fármaco aos brônquios, isto porque, ao disparar um jato do aerossol na aerocâmara as partículas aerossolizadas tendem a diminuir ainda mais de tamanho, facilitando a entrada do fármaco na via aérea inferior e, consequentemente, reduzindo as perdas no circuito do ventilador ${ }^{13,14,16,18}$. Embora não haja estudos de comparação entre diferentes tipos e tamanhos de aerocâmaras em VNI, é coerente conectar o pMDI com um espaçador próximo à máscara oronasal ${ }^{16}$.

Nava et al. ${ }^{10}$ em um estudo randomizado, placebo-controlado, investigaram a viabilidade da entrega de salbutamol com inalador pressurizado durante VNI em pacientes com DPOC. A resposta clínica foi medida através de testes de função pulmonar e o estudo mostrou que houve um aumento significativo do volume expiratório forçado no primeiro segundo $\left(V_{E F}\right)$ e da capacidade vital forçada (CVF) quando comparado com a administração de placebo acoplado à aerocâmara. Este é um dos principais estudos que mostram a efetividade da administração de broncodilatadores durante a VNI.

\section{FATORES RELACIONADOS AO VENTILADOR MECÂNICO}

\section{Portas de vazamento e disparo do jato}

Branconnier e Hess ${ }^{15}$ avaliaram a entrega de aerossol, utilizando salbutamol $100 \mu \mathrm{g}$ durante VNI para comparar os efeitos na deposição pulmonar do fármaco quando as portas de vazamentos (escape) eram incorporadas na máscara oronasal ou no circuito. Eles utilizaram um estudo experimental com modelo pulmonar simulando respiração espontânea, com volume corrente de $400 \mathrm{~mL}$ e dois níveis de pressão configurados, mimetizando um BILEVEL com IPAP $15 \mathrm{cmH}_{2} \mathrm{O}$ e EPAP $5 \mathrm{cmH}_{2} \mathrm{O}$ - pressão inspiratória e pressão expiratória positiva na via aérea, respectivamente. Um filtro foi acoplado ao modelo pulmonar e, posteriormente, analisado e quantificado o fármaco presente no pulmão artificial. Para este ensaio, um espaçador foi colocado entre a máscara e o circuito, como mostrado na Figura 1, e quatro jatos do fármaco foram disparados com intervalo de 15 segundos entre eles, sincronizando cada jato disparado com o início da inspiração ou com o início da expiração.

Os achados deste estudo mostraram que não há diferença na eficácia da entrega do aerossol quando o escape é incorporado na máscara ou no circuito $(P=0,71)$. Contudo, o estudo mostrou que a entrega de salbutamol foi significativamente menor quando este foi disparado na fase expiratória. A dose de fármaco entregue após quatro atuações, foi de aproximadamente $35 \mu \mathrm{g}$ durante a inspiração, enquanto na fase expiratória, a entrega foi em torno de $15 \mu \mathrm{g}(P<0,001)$.

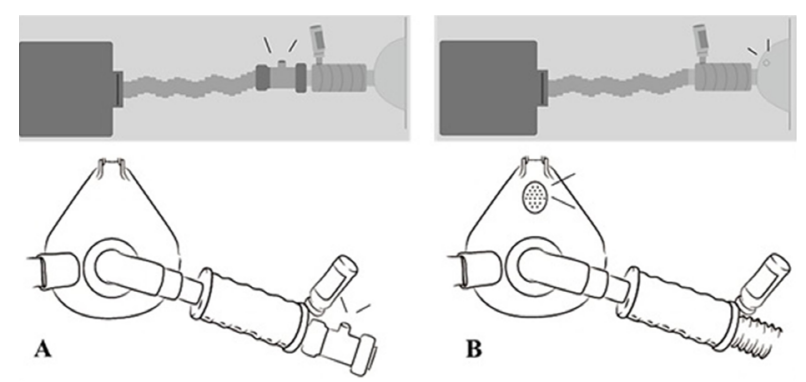

Figura 1: Visão lateral e frontal da conexão da aerocâmara no circuito de VNI. A: pMDI acoplado à aerocâmara e escape de $\mathrm{CO}_{2}$ no circuito; $\mathrm{B}$ : pMDI acoplado à aerocâmara e escape de $\mathrm{CO}_{2}$ na máscara oronasal.

Hassan et al. ${ }^{11}$, em um estudo experimental, fizeram uma comparação entre dois dispositivos geradores de aerossol: nebulizadores e inalador pressurizado acoplado a uma aerocâmara, ambos com salbutamol. Eles utilizaram um modelo pulmonar conectado 
a um filtro para posterior quantificação, simulando respiração espontânea com dois níveis de pressão configurados: IPAP $20 \mathrm{cmH}_{2} \mathrm{O}$ e EPAP $5 \mathrm{cmH}_{2} \mathrm{O}$; volume corrente de $500 \mathrm{~mL}$ e taxa respiratória de 15 respirações/minuto. $O$ estudo mostrou que para o pMDI, o disparo do jato foi sincronizado com o começo da inspiração e a porta de vazamento incorporada ao circuito. O resultado comparativo apontou que $2 \mathrm{mg}$ entregues a partir de pMDI com aerocâmara seria equivalente de 3 a $4 \mathrm{mg}$ entregues com nebulizador. Esta diferença na entrega de aerossóis nos dois métodos de inalação ressaltam a necessidade de ajuste de dose ao substituir um dispositivo de inalação por outro e que há perdas no sistema.

Dhand $^{14,16,18}$, responsável por inúmeros estudos a respeito do uso de dispositivos inalatórios em ventilação mecânica, sugere o uso de pMDI acoplado à aerocâmara; inserção de válvulas de exalação ou máscara com escape para contornar a reinalação de $\mathrm{CO}_{2}$ e disparo do jato sincronizado com o início da fase inspiratória.

\section{Parâmetros do aparelho}

Dhand ${ }^{16}$ mostra que a aplicação de pressão positiva na via aérea permite uma redução do tamanho de partícula do aerossol, aumento do volume corrente e diminuição da frequência respiratória, possibilitando uma maior entrega do fármaco à via aérea inferior. Altas taxas de fluxo de ar não são recomendadas na VNI pois causam aumento da resistência da via aérea e ressecamento das mucosas oral e nasal. Assim, a umidificação deve ser empregada para que o gás inalado possa gerar maior conforto ao paciente e, consequentemente, melhor adaptação à interface.

O efeito da umidificação do circuito não é bem estabelecido para VNI ${ }^{17}$. Durante VMI, a entrega de fármaco à via aérea inferior é reduzida $40 \%$ quando comparado com um circuito seco $(P<0,01)$. Aumidade do circuito provavelmente interfere no tamanho de partícula do fármaco, aumentando as perdas, podendo pressupor que os efeitos da umidade do circuito sobre a entrega do aerossol são semelhantes, tanto na VMI quanto na $\mathrm{VNI}^{16}$. Apesar dos efeitos indesejáveis da umidade na entrega do fármaco, a remoção do umidificador não é recomendada para a terapia de rotina com aerossóis, visto que, requer a interrupção do circuito e a espera de alguns minutos para que ele seque, além de causar desconforto ao paciente ${ }^{16}$.

\section{Interface}

Quatro estudos recomendaram a minimização de vazamentos indesejáveis de ar, certificando-se que a interface esteja corretamente posicionada no rosto do paciente e bem vedada. Esses estudos aconselham que a máscara oronasal esteja justa ao rosto do paciente para evitar vazamentos ou escape das partículas aerossolizadas do fármaco para a região dos olhos ${ }^{12,13,16,17}$.

\section{FATORES RELACIONADOS AO PACIENTE}

\section{Tipo de obstrução da via aérea}

O tipo, a gravidade e o mecanismo de obstrução da via aérea são fatores que influenciam na entrega do medicamento aerossol. Da mesma forma, a assincronia paciente-ventilador e a presença de hiperinsuflação dinâmica afetam a deposição do aerossol'13,18. Também se recomenda que a administração do medicamento seja feita, preferencialmente, com a posição de cabeceira elevada, uma vez que a posição sentada melhora a oferta do medicamento ${ }^{13}$.

\section{Tolerabilidade ao suporte}

A tolerabilidade em suportar a máscara oronasal é um critério importante para o sucesso da VNI. A máscara aumenta a dificuldade ao falar, da mesma forma que gera uma sensação de claustrofobia, sendo importante a adaptação do paciente a ela. Além disso, o fluxo de ar constante também interfere no conforto e na sincronia do paciente com o aparelho ${ }^{12,13,16}$.

As consultas às sociedades médicas resultaram em dois documentos que mencionavam sobre a administração inalatória de fármacos durante a VNI. A Diretriz Brasileira de Ventilação Mecânica de $2013^{8}$ sugere que a administração do pMDI seja feita de forma acoplada a um espaçador e enfatiza que o inalador pressurizado apresenta vantagens em relação à nebulização por ter maior facilidade de manipulação, reprodutibilidade de dose e menor risco de contaminação. A diretriz sugere que quando administrado $\beta 2$-agonistas adrenérgicos deve-se usar a dose de 4 jatos inicialmente com intervalo de 20 minutos até 3 vezes e, no tratamento de manutenção da DPOC, a cada 2-4 horas.

O segundo documento é um relatório da European Respiratory Society, que recomenda a aerossolterapia utilizando pMDI acoplado a um espaçador. O relatório sugere uma técnica de administração que consiste em: minimizar vazamentos na máscara e no circuito; utilizar espaçador cilíndrico, de aproximadamente $140 \mathrm{~mL}$ entre o circuito e a máscara; agitar o pMDI e acoplá-lo à aerocâmara; acionar o pMDI no início da fase inspiratória; aguardar 15 segundos para repetir novos disparos e monitorar o paciente quanto a resposta terapêutica ${ }^{19}$.

Não foram encontradas recomendações relacionadas à técnica inalatória durante VNI nas bulas e monografias dos fármacos selecionados ${ }^{20-29}$. Das oito empresas contatadas, quatro responderam aos nossos questionamentos, como mostrado na Tabela 1. 
Tabela 1: Respostas das empresas através do SAC.

\begin{tabular}{|c|c|}
\hline Empresas Contatadas & Respostas \\
\hline \multicolumn{2}{|c|}{ Fornecedoras de Ventiladores } \\
\hline Fornecedora A & Indicou buscar orientações com médico especialista. \\
\hline Fornecedora B & Sem resposta. \\
\hline Fornecedora C & Sem resposta. \\
\hline \multicolumn{2}{|l|}{ Indústrias Farmacêuticas } \\
\hline Indústria A & $\begin{array}{l}\text { Acoplar ao circuito de VNI uma aerocâmara com pMDI ou, caso } \\
\text { a situação clínica permita, retirar a VNI do paciente e administrar pela } \\
\text { via inalatória diretamente na cavidade oral com uso de aerocâmara } \\
\text { acoplada ao pMDI. }\end{array}$ \\
\hline Indústria B & $\begin{array}{l}\text { Empresa realizou uma busca de literatura, mas não recomendou o uso } \\
\text { do medicamento de maneira inconsistente com sua rotulagem aprovada. } \\
\text { Empresa realizou uma busca na literatura e não encontrou relatos de }\end{array}$ \\
\hline Indústria C & $\begin{array}{l}\text { uso do medicamento produzidos por eles a pacientes em VNI, portanto, } \\
\text { não indicam o uso concomitante ao suporte ventilatório. }\end{array}$ \\
\hline \multicolumn{2}{|c|}{ Fabricantes de Aerocâmaras } \\
\hline Fabricante A & Sem resposta. \\
\hline Fabricante B & $\begin{array}{l}\text { Recomendou que aerocâmaras projetadas para ventilação mecânica } \\
\text { devem ser acopladas no ramo inspiratório do circuito. }\end{array}$ \\
\hline
\end{tabular}

pMDI: pressurized metered dose inhaler, VNI: ventilação não-invasiva.

\section{DISCUSSÃO}

A VNI é um tratamento não-farmacológico recomendado na exacerbação da DPOC, assim como o uso de broncodilatadores é indicado como farmacoterapia no manejo da doença. No entanto, com base nos achados desta pesquisa podemos inferir que múltiplos são os fatores que influenciam na terapia inalatória, o que torna a administração complexa neste contexto.

A indisponibilidade de informações técnicas em bulas e monografias assim como as poucas informações disponíveis nas sociedades médicas demonstra a dificuldade em relação à técnica inalatória nesta condição. Além disso, ao contatar as empresas, pode-se notar a dificuldade das próprias produtoras de aerocâmaras especiais em se responsabilizar por uma orientação mais detalhada sobre as técnicas de utilização do aerossol. Na prática habitual de administração desses fármacos, a técnica se faz desconectando o suporte do paciente e realizando a inalação diretamente pela cavidade oral ${ }^{13,16,30}$. Essa forma comum de administração foi uma das recomendações fornecidas por uma das empresas contatadas.

É consenso entre os autores que as portas de vazamento devem ser incorporadas no circuito ou na máscara e que o disparo do jato deve ser feito, precisamente, com o início da fase inspiratória. Em casos de circuitos de membro-duplo - àqueles com dois tubos flexíveis: um para fase inspiratória e outro para fase expiratória - o pMDI deve ser acoplado no tubo responsável pela incursão inspiratória. Todos os estudos recomendam o uso de pMDI acoplado a uma aerocâmara, sendo esta específica para o encaixe no circuito ${ }^{7,10-19}$.

A principal dificuldade em relação às aerocâmaras é a aquisição deste material, já que, poucas são as opções no mercado brasileiro, além do valor agregado alto e recomendação do fornecedor para descarte a cada 24 horas. Outra opção para aquisição é a importação, porém, uma das produtoras de aerocâmaras especiais não realiza vendas para fora do país de origem. A conexão do pMDI ao circuito na forma como o dispomos, ou seja, naqueles casos em que há ausência de uma aerocâmara específica é uma demanda importante a ser estudada, assim como é imprescindível que haja treinamento adequado às equipes assistenciais para a correta conexão da aerocâmara ao ventilador e técnica de inalação.

Outra questão a ser discutida é em relação às configurações ideais para os parâmetros do aparelho. Umidade é um fator que diminui a entrega do fármaco aos pulmões, mas em contrapartida, não pode ser retirada visto o risco de dano às mucosas. Idealmente, seria necessário definir a umidade adequada para administração destes fármacos, determinando a umidade suficiente para gerar conforto ao paciente com menor perda de dose no circuito.

Alguns dos estudos selecionados recomendam determinadas configurações dos parâmetros do aparelho, contudo, salientam que elas ainda não estão bem estabelecidas ${ }^{11,12,14-17}$. A sugestão é que as configurações possam ser ajustadas no ato da administração do medicamento, se a condição clínica permitir e após, retornar aos parâmetros previamente configurados para o paciente. As configurações em comum entre os estudos foram: volume corrente próximo 
a $500 \mathrm{~mL}$; taxa respiratória de 15-20 respirações/ minuto; IPAP de $15-20 \mathrm{cmH}_{2} \mathrm{O}$ e EPAP $5 \mathrm{cmH}_{2} \mathrm{O}$.

A dificuldade em identificar parâmetros ideais do aparelho e maiores detalhes sobre o perfil de entrega do fármaco à via aérea inferior são limitações deste trabalho, sendo necessários estudos que quantifiquem a perda total de dose no circuito e, consequentemente, estabeleça uma adequação de posologia para administração em VNI. Testes de avaliação da função pulmonar obtidos pela espirometria, tais quais $\mathrm{VEF}_{1} \mathrm{e}$ CVF, antes e após a administração do medicamento podem ser utilizados para avaliar a eficiência da entrega do aerossol durante a $\mathrm{VNI}^{10}$. Um parâmetro inespecífico, mas que também pode ser avaliado, é a frequência cardíaca já que os $\beta 2$-agonistas adrenérgicos têm atividade nos receptores cardíacos. Outra maneira possível é utilizar os parâmetros contidos no ventilador para estimar complacência pulmonar e resistência da via aérea pré e pós uso do broncodilatador ${ }^{31}$. Em termos de pesquisa, pode-se realizar dosagem de salbutamol excretado na urina ${ }^{11}$ e emprego de aerossóis radiomarcados ${ }^{32}$.

A essa discussão inclui-se a multidisciplinaridade desse trabalho visto a abrangência desta condição especial de administração de fármacos inalatórios. Para uma avaliação fidedigna e integral, levando em consideração os diferentes saberes de cada área, é imprescindível uma discussão entre médico especialista, fisioterapeuta, farmacêutico e equipe de enfermagem.

\section{CONCLUSÃO}

A ventilação não-invasiva é uma modalidade de tratamento cada vez mais utilizada em pacientes com DPOC, uma vez que demonstrou evitar diversas complicações de maior gravidade, como intubação endotraqueal. Sabendo-se da importância da continuidade da terapia inalatória durante esse tipo de suporte e dadas evidências de desconhecimento sobre as melhores práticas ou técnicas de administração inalatória nesse contexto, reuniu-se os principais achados científicos sobre os fatores que afetam a aerossolterapia durante a VNI.

Podemos compreender que a oferta de fármacos nesta situação é complexa e múltiplos fatores são reconhecidos como interferentes na melhora da deposição pulmonar. É importante atentar para as recomendações que podem melhorar a deposição do fármaco na via aérea inferior, como uso de aerocâmara acoplada ao pMDI, minimização de escape indesejável de ar, sincronia entre pacienteventilador, disparo do jato na fase inspiratória, inserção de portas de vazamento no circuito ou na máscara e adequação dos parâmetros ventilatórios durante a administração.

\section{Agradecimentos}

Agradecemos ao Hospital de Clínicas de Porto Alegre, aos diversos profissionais de saúde envolvidos nas discussões sobre técnica inalatória em condição especial, especialmente aos fisioterapeutas, e à Michele Barreto pela ilustração das figuras de apoio à compreensão do texto.

\section{Conflitos de interesse}

Os autores declaram não haver conflito de interesse.

\section{REFERÊNCIAS}

1. Global Initiative for Chronic Obstructive Lung Disease. Global strategy for the diagnosis, management, and prevention of chronic obstructive pulmonary disease (2020 report) [Internet]. Fontana (WI): GOLD; 2020 [citado 20 nov 2020]. Disponível em: https://goldcopd.org/wp-content/ uploads/2019/12/GOLD-2020-FINALver1.2-03Dec19_WMV.pdf

2. Brasil. Ministério da Saúde. Protocolo clínico e diretrizes terapêuticas: doença pulmonar obstrutiva crônica. Brasília, DF; 2013.

3. Correia S, Luz F, Amaral V, Dias A, Miragaia T. Avaliação do conhecimento sobre a utilização de inaladores entre médicos e profissionais de farmácia dos Açores. Rev Port Med Geral Fam. 2015;31(1):14-22.

4. Maciel PP. Efeito da intervenção educativa sobre a técnica de administração de medicamentos inalatórios por profissionais de enfermagem [trabalho de conclusão de curso]. Porto Alegre: Universidade Federal do Rio Grande do Sul; 2009.

5. Muchão FP, Perín SRR,

Rodrigues JC, Leone C, Silva Filho LVRF. Evaluation of the knowledge of health professionals at a pediatric hospital regarding the use of metered-dose inhalers. $J$ Bras Pneumol. 2008;34(1):4-12.
6. Ram FSF, Picot J, Lightowler J, Wedzicha JA. Non-invasive positive pressure ventilation for treatment of respiratory failure due to exacerbations of chronic obstructive pulmonary disease. Cochrane Database Syst Rev. 2003;(4):CD004104.

7. Keenan SP, Sinuff T, Cook DJ, Hill NS. Which patients with acute exacerbation of chronic obstructive pulmonary disease benefit from noninvasive positive-pressure ventilation? A systematic review of the literature. Ann Intern Med. 2003;138(11):861-70. 
8. Associação de Medicina Intensiva Brasileira; Sociedade Brasileira de Pneumologia e Tisiologia. Diretrizes Brasileiras de Ventilação Mecânica. São Paulo: AMIB; 2013.

9. Souza MT, Silva MD, Carvalho R. Revisão integrativa: o que é e como fazer? Einstein (Sao Paulo). 2010;8(1):102-6.

10. Nava S, Karakurt S, Rampulla C, Braschi A, Fanfulla F. Salbutamol delivery during non-invasive mechanical ventilation in patients with chronic obstructive pulmonary disease: a randomized, controlled study. Intensive Care Med. 2001;27(10):1627-35.

11. Hassan A, Salah Eldin R, Abdelrahman MM, Abdelrahim ME. In-vitro/in-vivo comparison of inhaled salbutamol dose delivered by jet nebulizer, vibrating mesh nebulizer and metered dose inhaler with spacer during non-invasive ventilation. Exp Lung Res. 2017;43(1):19-28.

12. Rzepka-Wrona P, Skoczynski S, Wrona D, Barczyk A. Inhalation Techniques Used in Patients with Respiratory Failure Treated with Noninvasive Mechanical Ventilation. Can Respir J. 2018;2018:8959370.

13. Maccari JG, Teixeira C, Gazzana MB, Savi A, Dexheimer-Neto FL, Knorst MM. Terapia inalatória em ventilação mecânica. J Bras Pneumol. 2015;41(5):467-72.

14. Dhand R. Basic Techniques for Aerosol Delivery During Mechanical Ventilation. Respir Care. 2004;49(6):611-22.
15. Branconnier MP, Hess DR. Albuterol delivery during noninvasive ventilation. Respir Care. 2005;50(12):1649-53.

16. Dhand R. Aerosol Therapy in Patients Receiving Noninvasive Positive Pressure Ventilation. J Aerosol Med Pulm Drug Deliv. 2012;25(2):63-78.

17. Hess DR. Aerosol Therapy During Noninvasive Ventilation or HighFlow Nasal Cannula. Respir Care. 2015;60(6):880-93.

18. Dhand R. Aerosol Delivery During Mechanical Ventilation: From Basic Techniques to New Devices. J Aerosol Med Pulm Drug Deliv. 2008;21(1):45-60.

19. Laube $B$, Janssens $H$, De Jongh $F$, Devadason S, Dhand R, Diot P, et al. What the pulmonary specialist should know about the new inhalation therapies. Eur Respir J. 2011;37(6):1308-31.

20. GlaxoSmithKline. Aerolin ${ }^{\circledR}$ spray [Internet]. Rio de Janeiro; 2021 [citado 20 abr 2021]. Disponível em: https://br.gsk.com/media/535927/ rec_1501210707324505697_bl_ aerolin_aer_gds24ipi08_10418.pdf

21. Boehringer Ingelheim. Duovent $N \circledast$ [Internet]. Itapecerica da Serra; 2020 [citado 20 abr 2021] Disponível em: https://www.boehringer-ingelheim. com.br/sites/br/files/duovent_n_paciente_profi.pdf

22. AstraZeneca. Vannair $[$ [Internet]. Cotia; 2020 [citado 20 abr 2021] Disponível em: https://www.astrazeneca.com.br/ content/dam/az-br/Medicine/medicinepdf/Vannair\%20-\%20VAN011\%20-\%20 Bula\%20Paciente.pdf
23. Budesonide and formoterol. Waltham (MA): UpToDate; 2019.

24. Albuterol. Waltham (MA): UpToDate; 2019.

25. Ipratropium. Waltham (MA): UpToDate; 2019.

26. Ipratropium. Greenwood Village (CO): IBM Corporation; 2019.

27. Albuterol. Greenwood Village (CO): IBM Corporation; 2019.

28. Budesonide. Greenwood Village (CO): IBM Corporation; 2019.

29. Fenoterol. Greenwood Village (CO): IBM Corporation; 2019.

30. Asturian K, Ferreira MAP. Administração de medicamentos inalatórios durante ventilação não-invasiva e/ou traqueostomia. Rev Bras Farm Hosp Serv Saude. 2020;11(1):0372.

31. Mouloudi E, Katsanoulas K, Anastasaki M, Hoing S, Georgopoulos D. Bronchodilator delivery by metered-dose inhaler in mechanically ventilated COPD patients: influence of tidal volume. Intensive Care Med. 1999;25(11):1215-21.

32. Galindo-Filho VC, Alcoforado L, Rattes C, Paiva DN, Brandão SCS, Fink JB, et al. A mesh nebulizer is more effective than jet nebulizer to nebulize bronchodilators during noninvasive ventilation of subjects with COPD: A randomized controlled trial with radiolabeled aerosols. Respir Med. 2019;153:60-7. 